139-189.

HOOKER, J. D. -1873-Curtis's Botanical Magazine. $3^{a}$ serie. Vol. 29. L. Reeve \& CO., 5, Henrietta Street Covent Garden, London.

JAHANDIEZ, É. \& R. MAIRE - 1934- Catalogue des Plantes du Maroc (Spermatophytes et Ptéridophytes). Vol. III. Imprimerie Minerva, Alger.

KUNKEL, G. -1980- An excursion through my herbarium. II. Vieraea 8(2): 342-345

LOWE, R. T. -1872- Manual flora of Madeira and the Adjacent islands of Porto Santo and the Desertas. Vol. 1. John van Voorst, 1, Paternoster row (Printed by Taylor and Francis, Red Lion Court, Fleet-Street), London.

MAIRE, R. C. J. E. -1927- Contributions à l'Étude de la Flore de l'Afrique du Nord. Mémoires de la Société des Sciences Naturelles du Maroc. Rabat, Morocco 15(11): 45-48

MAIRE, R. -1928-Contributions à l'Étude de la Flore de 1'Afrique du Nord. Bulletin de la Société d'Histoire Naturelle de l'Afrique du Nord 19(12): 58-59

Authors addresses. ${ }^{1}$ GBM, Universidade da Madeira, Centro de Ciências da Vida, Campus da Penteada, 9020-105 Funchal - Portugal. ${ }^{2}$ Real Jardín Botánico, CSIC, Plaza de Murillo, 2, 28014-Madrid, España. ${ }^{3}$ Institute of Botany, Academy of Sciences of the Czech Republic, Zámek 1, 252 43, Průhonice, Czech Republic. ${ }^{4}$ Department of Botany, Faculty of Science, Charles University in Prague, Benátská 2, 12801 Prague, Czech Republic. *Author for correspondence: mzitaferreira@sapo.pt

22. NUEVO NOMBRE EN RHODANTHEMUM (COMPOSITAE, ANTHEMIDEAE).

Francisco GÓMIZ GARCÍA

Recibido el 26 de mayo de 2014, acetado para su publicación el 30 de junio de 2014

New name in Rhodanthemum (Compositae, Anthemideae).

Palabras clave. Rhodanthemum redieri subsp. soriae, Leucanthemum redieri var. cuneatum, Flora, Taxonomía, Marruecos, Norte de África

Key words. Rhodanthemum redieri subsp. soriae, Leucanthemum redieri var. cuneatum, Flora, Taxonomy, Morocco, North Africa.

Rhodanthemum (Vogt) Wilcox, Bremer \& Humphries, es un género perteneciente a la tribu Anthemideae, subtribu Leucantheminae, (Compositae), cuyo área de distribución se extiende por el norte de África (Argelia y Marruecos) y el sudoeste de Europa (España)
(Bremer \& Humphries, 1993) (Oberprieler, Himmelreich \& Vogt, 2007).

En Marruecos está representado por una docena de especies endémicas, más otras dos de distribución argelino-marroquí y una más hispano-marroquí (Ibn Tattou \& Fennane, 


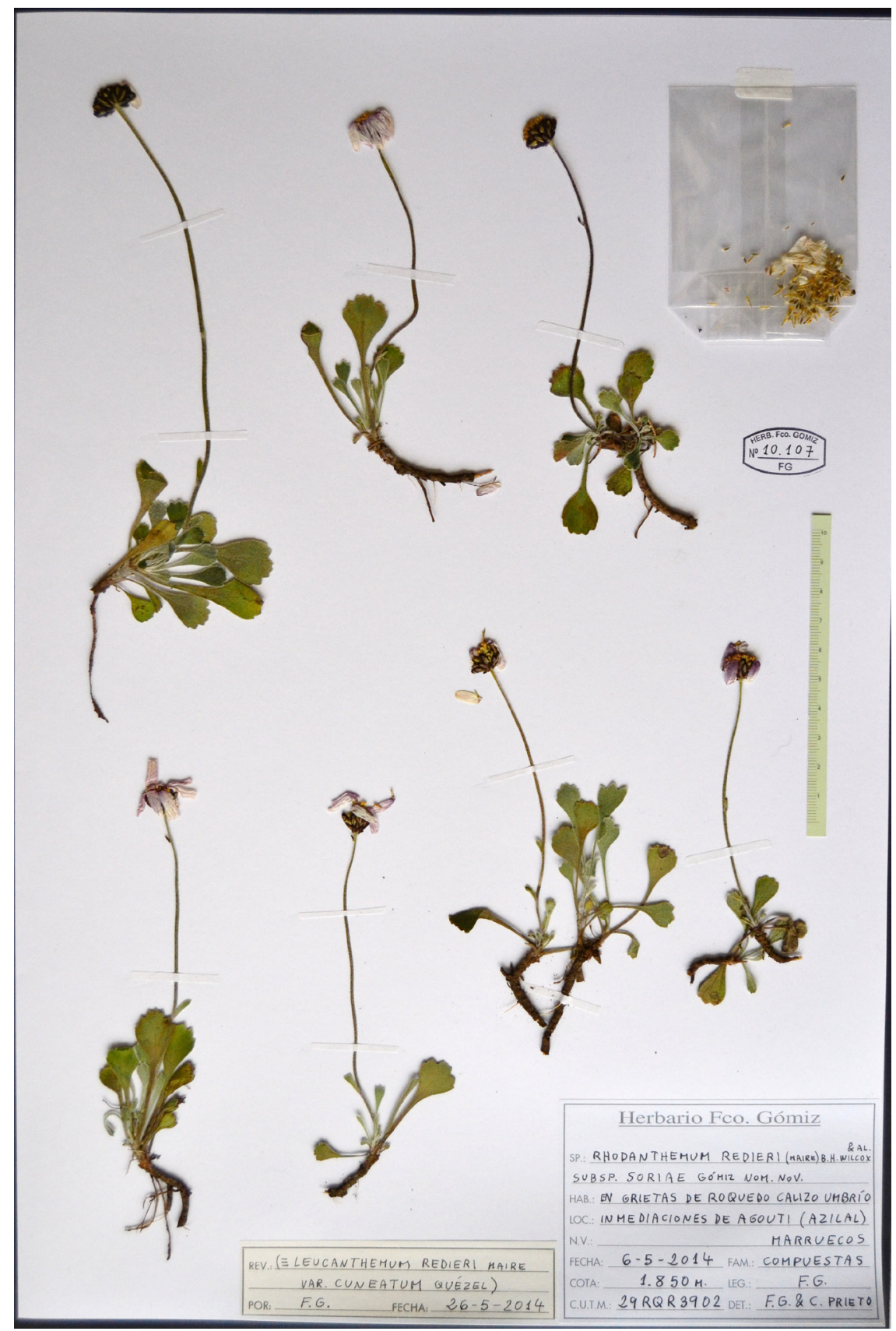

Figura 1. Pliego de Rh. redieri subsp. soriae. [Transcripción de las etiquetas: Herbario Fco. Gómiz / Sp.: Rhodanthemum redieri (Maire) B. H. Wilcox \& al. / subsp. soriae Gómiz nom.nov. / Hab.: en grietas de roquedo calizo umbrío / Loc.: inmediaciones de Agouti (Azilal) MARRUECOS / Fecha: 6-5-2014 Fam.: Compuestas / Cota: 1.850 m. Leg.: F. G. \& C. Prieto / C.U.T.M.: 29R QR 3902 Det.: F. G. /// Rev.: (三 Leucanthemum redieri Maire / var. cuneatum Quézel) / Por.: F. G. Fecha: 26-5-2014]. 
2008).

En las dos últimas prospecciones llevadas a cabo en la región del M'Goun (Alto Atlas), en junio de 2013 y mayo de 2014, encontramos frente a Agouti, en el valle de Aït Bouguemez, unas poblaciones de Rhodanthemum, cuyos individuos parecían, a primera vista, muy diferentes a los de cualquier otra especie conocida de dicho género. Tras la revisión de la correspondiente bibliografía y de los especímenes de herbario que nos ha sido posible consultar, se ha podido comprobar que dicho material se puede asimilar a Leucanthemum redieri Maire var. cuneatum Quézel. El carácter más llamativo de estas plantas reside en sus hojas que a diferencia de todas las demás especies del género, que las presentan profundamente divididas en más o menos estrechos lóbulos, en este caso son enteras o subenteras y rematadas en 3-7 dientes, lo que les confiere un cierto aspecto entre cuneiforme y flabelado. El material tipo de Quézel, depositado en RAB, es escaso y fragmentado, pero tras el estudio morfológico del más abundante recogido ahora, y su comparación con los demás tipos nomenclaturales del género, creemos que se trata de una buena subespecie que dedicamos a Pedro Soria Esteván, discípulo del Hno. Rufino Sagredo y coautor de la Flora Vascular de Andalucía Oriental.

Rhodanthemum redieri (Maire)B. H. Wilcox $\&$ al. subsp. soriae Gómiz, nom. nov.

$\equiv$ Leucanthemum redieri Maire var. cuneatum Quézel in Bull. Soc. Sci. Nat. Phys. Maroc, 34: 314 (1954) [syn. subst.]

Tipus: "M'Goun: gorges de l'acif Isladen, 2.600 m. Quézel, P. 1952, RAB 34976”.

\section{Material estudiado}

MARRUECOS. M'GOUN: Gorges de l'acif Isladen, 2.600 m., Quézel, P. 1952, RAB 34976; Inmediaciones de Agouti (Azilal), 29RQR3902, $1.850 \mathrm{~m}$, en grietas de roquedo calizo umbrío, 31 37'N 6²8'W, 14-VI-2013, Fco.Gómiz, herb.Fco.Gómiz, FG 9837; ibid., $1.820 \mathrm{~m}, 31^{\circ} 37^{\prime} 44^{\prime \prime} \mathrm{N} 6^{\circ} 28^{\prime} 44^{\prime \prime} \mathrm{W}$, 6-V-2014, Fco. Gómiz \& C. Prieto, MGC 79437, MA-883456, BC-934889, herb. Fco. Gómiz, FG 10107 y FG 10108.

\section{Observaciones}

Rhodanthemum redieri subsp. soriae se diferencia de las otras dos subespecies de Rhodanthemum redieri -subsp. redieri y subsp. humbertii Gómiz-, por presentar unos limbos foliares largamente atenuados en cuña y mucho menos divididos; los ápices de los dientes foliares carecen del característico pincel de pelos; y los capítulos son algo menores en todas sus partes - lo que no concuerda demasiado con la diagnosis de Quezel que afirma que las lígulas son mayores en su var. cuneatum-. Otra peculiaridad de esta planta consiste en la desarticulación que se produce, con frecuencia, en sus pelos foliares: estos son basifijos pero con una articulación basal, de forma que, cuando se desprende el flagelo superior de casi un milímetro de longitud, ciertas zonas de la superficie foliar, o toda ella, presentan un cierto aspecto alampiñado y papilar.

La planta que nos ocupa, presenta una fenología muy temprana -teniendo en cuenta que ocupa roquedos umbríos orientados al norte y que medra por encima de los $1.800 \mathrm{~m}$-: en junio de 2013 encontramos las plantas con los receptáculos totalmente desprovistos de semillas, es decir, con la diáspora completamente finalizada; decididos a encontrar individuos en floración adelantamos la visita cinco semanas en 2014, comprobando con sorpresa que la floración estaba bastante avanzada, con las lígulas muy reflejas y algo marchitas en los capítulos que aún no las habían perdido, lo que nos ha permitido recoger cipselas maduras a punto de dispersarse; deducimos, pues, que la floración debe producirse a primeros de abril, o incluso antes, lo que podría haber hecho 


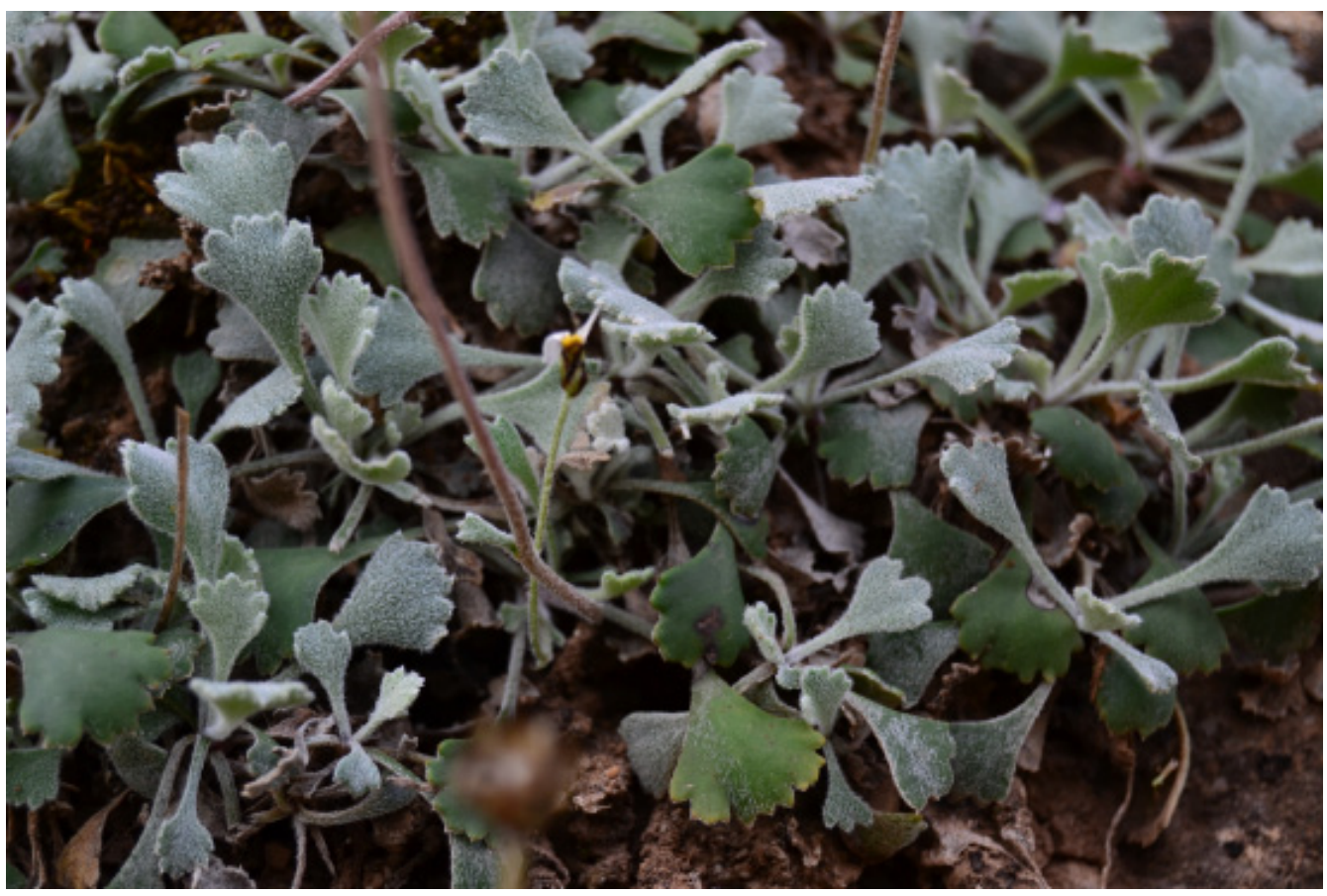

Figura 2. Detalle de un grupo compacto de rosetas foliares mostrando el característico aspecto de las hojas de $R h$. redieri subsp. soriae

pasar inadvertidas estas plantas, hasta ahora, al ser ese un mes muy temprano para herborizar en la región del M'Goun. El material tipo de Quezel, conservado en RAB, presenta un único capítulo que podría corresponder a una planta con floración extemporánea.

Como ocurre con otras especies del género, Rhodanthemun redieri subsp. soriae ocupa grietas y anfractuosidades de roquedos, fuera del alcance del ganado. En esta segunda visita, compartía hábitat con otra asterácea de porte parecido y algo más abundante: Bellis caerulescens Ball, también endémica de los Atlas.
Bull. nat. Hist. Mus. Lond. (Bot.) 23(2):71-177. IBN TATTOU, M. \& M. FENNANE -2008- Flore vasculaire du Maroc: inventaire et chorologie (Vol. 2). Trav. Inst. Sci., Sér. Bot. 39:1-398.

OBERPRIELER, C., S. HIMMELREICH \& R.VOGT -2007-A new subtribal classification of the tribe Anthemideae (Compositae). Willdenowia 37:89-114.

VOGT, R. -2005- Lectotypification of twenty-one names in Rhodanthemum (Compositae, Anthemideae). Taxon 54(2):479-484.

\section{BIBLIOGRAFÍA}

BREMER, K. \& C.J. HUMPHRIES -1993- Generic monograph of the Asteraceae-Anthemideae.
Dirección del autor: Apartado 1007, E-24080 León, España.fgomizgarcia@terra.com 